To appear in the Proceedings of ECSCW 97 Conference, Lancaster UK, September 1997.

\title{
Plans as Situated Action: An Activity Theory Approach to Workflow Systems
}

\author{
Jakob E. Bardram
}

Computer Science Department, Aarhus University and Kommunedata I/S, Denmark.

bardram@daimi.aau.dk

\begin{abstract}
Within the community of CSCW the notion and nature of workflow systems as prescriptions of human work has been debated and criticised. Based on the work of Suchman (1987) the notion of situated action has often been viewed as opposed to planning work. Plans, however, do play an essential role in realising work. Based on experiences from designing a computer system that supports the collaboration within a hospital, this paper discusses how plans themselves are made out of situated action, and in return are realised in situ. Thus, work can be characterised as situated planning. This understanding is backed up by Activity Theory, which emphasises the connection between plans and the contextual conditions for realising these plans in actual work.
\end{abstract}

\section{Introduction}

The issue of workflow systems has been addressed by several authors as ways of routing information objects among users, and to specify automatic actions to be taken in that routing typically according to certain process models (Medina-Mora et al., 1992; Abbott and Sarin, 1994; Schäl, 1996). A process model is typically understood as a computerised (i.e. formal) representation of work procedures that controls the order in which a sequence of tasks are to be performed. These workflow systems for the coordination of activities in organisations have drawn much attention, but have been subject to much controversy and criticism for their rigid representation of work in process models (Suchman, 1994; Winograd, 1994; Bowers et al., 1995; Heath and Luff, 1996). The potential danger with current workflow systems is that their design is predictated entirely by formal procedures ignoring (and even damaging) the informal practice (Symon et al., 1996).

Suchman (1987) shows the importance of differentiating between work and representations of work like 
plans and process models. Plans are representations of situated actions produced in the course of action and therefore they become resources for the work rather than they in any strong sense determine its course. Suchman emphasises action as essential situated and ad hoc improvisations, which consequently make plans rational anticipations, before the act, and post hoc reconstructions, afterward. The theoretical work on situated action, and the studies underlying it, seems to have attained so much attention that the importance of plans and protocols as guidance of work has been neglected. Recently, at the CSCW 96 conference in Boston, Suchman herself commented that an unfortunate, but typical, mis-reading of her work was that plans do not exist. Plans do exist and should be viewed as "an artifact of our reasoning about action, not ... the generative mechanism of action." (p. 39, emphasis in original).

Nevertheless, in medical work, pre-hoc representations of work like plans, checklists, schedules, protocols, work programmes etc. have proved extremely valuable as mechanisms giving order to work. Such plans support handling complex work situations, involving coordination and collaboration among several health professionals. For example, the patient s diagnosis and the associated treatment plan are essential coordination mechanisms, which convey information to the involved staff about the nature of the illness and how the treatment should proceed. Without this plan, extensive communication has to take place in order to inform all involved personnel about the patient, his illness and how the physician in charge intends to cure it. Thus, plans as pre-scriptions of activity are valuable, and indeed used, within organisations like hospitals to carry out work. This makes Schmidt and Simone (1996) raise the rhetoric question to Suchman of "What is it that makes plans such as production schedules, office procedures, classification schemes, etc. useful in the first place? What makes them resources ?" (p. 169).

These studies of work seem to leave us with what can be called the planning paradox: On the one hand, due to the contingencies of the concrete work situation work has an ad hoc nature. Plans are not the generative mechanisms of work, but are merely used to reflect on work, before or after. On the other hand, we find that plans, as more or less formal representations, play a fundamental role in almost any organisation by giving order to work and thereby they effectively help getting the work done. Within a hospital context this tension between informal practice and formal procedures for work is also discussed by Symon et al. (1996):

"[A]ny investigation of work coordination should look beyond formal procedures to consider contextual factors (i.e. factors that may give rise to informal practices), while at the same time taking into account the use and influence of formal procedures" (p. 3, emphasis in original).

This planning paradox is addressed in this paper. First, the theoretical understanding of human activity based on Activity Theory shows how a concept of planning does not necessarily mean total pre-handling and control of work, but can be achieved in the course of activity. The false dichotomy between plans and situated action is removed and it becomes possible to talk about, and thus support by computers, situated planning. This theoretical insight is then supported by empirical insight into the working of a Danish hospital by illustrating the important role, which planning plays within hospital work and how a computer system was designed to support planning without emphasising rigid matches between plans as representations of work and work itself. Finally, the paper concludes by arguing that a workflow system often exists in a tension between supporting a smooth flow of work within a work practice and the organisational needs for accounting for this work, and that this tension needs to be considered in design.

\section{Activity Theory}

Activity Theory originated in the former Soviet Union as part of the cultural-historical school of 
psychology founded by Vygotskij, Leontjev and Lurija. The theory is a philosophical framework for studying different forms of human praxis as developmental processes, with both the individual and social level interlinked. Within the HCI community, Activity Theory has recently attained increased attention (Bødker, 1991; Nardi, 1996) and has been proposed as a basis for CSCW research too (Kuutti, 1991). Here I will focus on certain core concepts of the theory, which are fundamental in understanding the role of technology and human activity as guided by plans. The following is based on the writing of Vygotskij (1978), Leontjev (1978; 1981), and Anokhin (1973; 1976).

The fundamental unit of analysis is the human activity which has three basic characteristics; firstly, it is directed towards a material or ideal object which distinguishes one activity from another; secondly, it is mediated by artifacts (tools, language, etc.); and thirdly, it is social within a culture. In this way, computer artifacts, like all other artifacts, mediate human activity within a practice. By acting in the world, human beings meet the objective world, which is experienced through the activity. Thus, human knowledge about the world is reflection obtained through activity, constituting the basis for expectations, and desires about activities in this world. This describes the basic dialectical relationship between the human being and the world, the subject and the object.

\section{The Structure and Development of Human Activity}

Human activity can be described as a hierarchy with three levels: activities realised through chains of actions, which are carried out through operations. Human activity is always directed toward a material or ideal object satisfying a need and the subject s reflection of, and expectation to, this object characterises the motive of the activity.

Human activity is carried out through actions, realising objective results. These actions are controlled by the subject's conscious goals, which are the anticipation of the future results of the action. The activity exists only as one or more actions but the activity and the action are not identical and cannot be reduced to each other. For example, for a physician the activity of diagnosing a patient can be realised in several ways. He can trust the diagnosis stated by the general practitioner on the referral papers. Or he can establish his own diagnosis by obtaining the necessary clinical data, like blood sugar level, X-ray pictures, etc, using the service departments at the hospital. Or he can use a computer-based patient record system to see if such data are already available. These are different actions, mediated by different tools, which all realise the activity of diagnosing the patient. On the other hand, the same action can be a part of realising different activities: The action of requesting an X-ray examination at the radiology department can be part of the diagnosing activity or it can be part of preparing for surgery, thus realising a total different activity. Furthermore, actions are usually polymotivated; two or more activities can temporarily merge, motivating the same action, if the goal is part of reaching the motives of several involved activities simultaneously.

Even though the goal of the action can be represented in the human mind independently of the situation in which it has to take place, the practical process of realising the action cannot be detached from the conditions of the concrete situation. Therefore, actions are realised through a series of operations; each accommodated to the concrete physical conditions of the action. While the analytical level of actions describes the intention of an activity what results should be obtained operations describe the operational level how the action is realised, adjusted to the actual material conditions of the action. For example, the way the phone is used to order an X-ray examination depends entirely on how the phone works, the phone number of the radiology department, the physical surroundings of the phone, etc. Operations are performed without thinking consciously but are oriented in the world by a non-conscious 
orienting basis of the operation. This orienting basis is established through experience with the concrete material conditions for the operation, and is a system of expectations about the execution of each operation controlling the operation, in the process of the activity. Again, the action and the operations realising the action are not identical and cannot be reduced to each other: an operation can be part of several actions (together with other operations) and the same action can be realised through different operations.

\section{Planning Recurrent Actions through Anticipatory Reflection}

At all three levels the human activity is guided by anticipation. This anticipation is the motive of the activity, the goal of the action and the orienting basis of the operation, respectively. The anticipation of future events is the fundamental principle of anticipatory reflection as developed by Anokhin. The classical example of anticipatory reflection is Anokhin s rethinking of Pavlov s discovery of the conditioned reflex: When a dog salivates in response to the ringing of a bell, it is not because saliva is needed to digest the bell but because the dog anticipates food to appear in the future which has to be digested. The anticipatory reflection guides the activity by making an afferent synthesis between a perception of the environmental state of the activity, and memory (i.e. the cumulated experience of the person). This afferent synthesis forms an anticipation of the future state as a result of the activity about to be performed. When the activity is performed there is a feedback mechanism which compares the result of the activity with the prediction, and any incongruence (i.e. a breakdown) gives rise to a learning situation (i.e. the experience of the person is expanded). This model of anticipatory reflection based on the afferent synthesis between perception and memory is a general model for all levels of the activity.

The basic principle that makes the anticipatory reflection possible is the recognition of recurrent structures in the world. The existing of all living beings and their reflection of recurrent structures, which repeat themselves over time, is the indispensable prerequisite for prediction. Pavlov s experiments also illustrate this because the response is mutually correlated with the amount of training sessions.

\section{Artifacts as Mediators and Crystallisation of Work}

Describing human activity as actions realised through operations helps to understand the fundamental role, which plans play in human cognition and activity. Based on prior experience the plan anticipates future results of the actions realising the activity, but these plans, or anticipations, have to be implemented through operations which are adjusted to the material conditions of the situation. The afferent synthesis explains how human activity indeed is planned, i.e. anticipated, and at the same time situated, i.e. contextual.

Now one could ask what plans, as cognitive constructs have to do with material artifacts like checklists, production lists and workflow systems? However, within the cultural-historical school there is no such differentiation between ideal (i.e. cognitive) and material artifacts: plans as artifacts are used to mediate activity regardless of whether they exist on e.g. paper or are memorised. Human work is characterised by the collaborative production of artifacts; each made with the purpose of mediating a certain activity. The mediating characteristics of an activity is therefore crystallised (or objectified) (Bærentsen, 1989) into these artifacts, and through use, the artifacts are continuously modified and shaped to meet the evolving human needs. For example, the radiology order form used at AAS is a product of years of experience in ordering X-ray examinations, containing fields that prompt for certain important information. Therefore, the cognitive plans and their material counterpart are mere reflections of each other because they are both resources for, and products of, human activity. 


\section{The SAIK project: Developing Computer Support for Clinical Work}

The SAIK project was launched as the experimental part of redesigning a national-wide mainframe-based Hospital Information System. The aim was to investigate the coordination and planning of patient care within hospitals and based on these investigations to develop a prototype called the Patient Scheduler illustrating how coordination of patient care within hospitals can be supported by computer technology.

This participatory design process took a 24-bed specialised medical (endocrinological) ward as point of departure for investigating the work and collaboration among departments within the hospital for the County of Aarhus (AAS). Typical patients at the ward are diabetics or elderly patients with osteoarthritis. AAS is a middle size Danish hospital with 1700 employees and 370 beds. It has 7 medical and surgical specialised departments, each with 2 - 4 wards, several out-patients clinics, and several service departments e.g. radiology, laboratory, and pathology. Historically, Danish hospitals, including AAS, have become increasingly specialised and centralised (Vallgårda, 1992). This has resulted in large hospitals with a large number of specialised departments. Because of this specialised nature of medical work, collaboration across departmental and professional borders is patient treatment and care per se, making the hospital an excellent place for investigating issues in computer support for people cooperation closely. For example, the daily treatment of all patients admitted to the ward is based on data from e.g. blood tests and X-ray pictures, which involves frequent communication and coordination with the laboratory and radiology departments, respectively.

A fundamental statement within the participatory design tradition is that a profound understanding of the users work practice is a pre-condition for designing computer support. This understanding of the work at AAS was done as workplace studies based on qualitative methods such as qualitative interviews; workshops; participative observations of daily work at the ward and service departments, meetings and conferences; and studies of different documents, records and other tools. Based on this understanding the Patient Scheduler was developed and used for further participatory design sessions at AAS. The Patient Scheduler aims at providing flexible support for requesting, booking and scheduling examinations, tests, etc. on different departments within the hospital.

\section{Planning as a Central Activity of Clinical Work}

Treatment of patients within a hospital can clearly be characterised as specialised and informal skills that have to take the contingencies of the concrete situation into account. Nevertheless, clinical work is subject to a large degree of planning and plans play a central role in guiding and recording work at a hospital. Let us consider three examples from the hospital: A central planning tool widely used within medical work is protocols of treatment, or Standard Operating Procedures (Strauss et al., 1985), which prescribe a standard treatment for a standard disease for a standard patient. Such protocols are developed by the clinical team who uses them, and they are supported by general policies and guidelines of use. A central part of such a protocol is often the unravelling program, which prescribes which initial examinations and tests should be ordered to state a precise diagnosis. Hence, the unravelling program provides a plan for obtaining the necessary clinical data for further treatment. Another planning tool applied at the ward is the 24-hour-care plan made every afternoon by the nurses on duty. This plan describes the care of each patient within the next 24 hours and functions as a "boundary object" (Star, 
1989) by carrying information between three working shifts in a standardised way. This plan is made according to the overall plan of treatment (the protocol) by taking into consideration the patient $\mathrm{s}$ condition in the concrete situation. By analysing the use of these planning tools from an Activity Theory perspective on CSCW, the following characteristics of plans emerged:

\section{Plans as Socially Constructed and Used Artifacts}

Documents used in daily work are socially constructed in and through the intersubjective understanding and use of members in a community. A document is not just a document, but a certain document like thr medical record (Hughes and King, 1993). Thus a certain document (record) is an artifact reflecting certain work activities and the socially defined purpose of these activities. For example, all departments within the hospital, like the medical, surgical and anaesthetic departments, have their own patient files and records, made to suit their special activities and needs. Similarly, plans are socially used and constructed as part of the ongoing work activities at the hospital. The production of the different unravelling plans used at the ward is an on-going activity closely connected to the treatment of patients. Thus, these plans are crystallisations of a historically developed socio-cultural knowledge of how to treat different kinds of diseases and patients. An implication of this is that plans and protocols change over time, and thus have a historicity. At the ward this is most evident in the continuous making of 24-hour-care plans by the nurses, but also unravelling plans and medical protocols for treatment of patients are changed to reflect the results of the latest research within the international medical community.

\section{The Difference between the Plan and the Instantiation of the Plan}

There is a fundamental distinction between a plan and an instantiation of the plan, i.e. the actual performance based on the plan. Building on prior experience, plans become resources, detached from the concrete and situated real-world activities, which later might implement and carry out the plan. The strength of the plan is the anticipation of future ways of performing activities, detached from, but still taking into account, the conditions of the real-world settings. When applying a plan to a concrete problem, the situated actions performed in the activity often mirror the plan, but are adjusted to the concrete details and conditions of the context. For example, the unravelling plan for an osteoarthritis patient might state that an X-ray image of the hip is necessary. But when applying the plan to Mr. Jones, who doesn t have any problems with his hips, this part of the plan may be skipped and other examinations, like a blood test, might be added to Mr. Jones unravelling plan. Thus instantiations of plans have fuzzy boundaries. When applying an unravelling plan at AAS, the actual use is reflected in the patient sexamination card that contains an overview of all examinations ordered or performed. Hence, the unravelling plan reflects the plan and the examination card reflects the instantiation of the plan.

\section{Plans as Means of Dividing Work}

Plans are used to organise the work, and when several people are involved in this work, the plan reflects the responsibility of the involved actors. Even if the plan does not contain a formal description of who is doing which part of the plan, this responsibility either refers to the wider organisational division of work or is clarified when the plan is instantiated. The nurses 24-hour-care plan, for example, is divided into sections that reveal the care to be undertaken by each workshift, thus explicitly reflecting the responsibility of each shift. On the other hand, when a medical protocol states that the temperature of a patient has to be measured twice a day, the protocol does not explicitly state who should do this, because 
this is the job of the nurse in charge of the particular patient within the particular workshift.

\section{Plans as Status Overviews}

As a result of carrying a division of labour, a plan works as a status overview, like a checklist, revealing the state of the work according to the prescribed plan. The characteristic of checking off items on a checklist becomes essential when several interdependent actors work together using plans to coordinate work. The 24-hour nursing plan helps coordinate the work across working shifts because the different tasks listed in it are marked done when performed. Similarly, the examination card reflects the status of the unravelling programme of a patient, containing information on the status of each test, whether they are prescribed, ordered, or carried out.

\section{Plans as Records}

Often when plans are used in work settings, like a hospital, the interesting issue is not to follow the plan but the deviation from the plan. Deviating from a plan is a breakdown and therefore a potential learning situation. This fact is well recognised within medical work, where the use of problem-oriented records is becoming more widespread. Problem-oriented records are based on general medical protocols for treatment of a disease, like diabetes or appendicitis, and when a patient is treated, only deviations from this protocol are recorded. This makes problem-oriented records very powerful tools, because they contain only potential learning material compared to the standard protocol and, at the same time, they are extremely effective in both production and use.

\section{The Patient Scheduler}

The Patient Scheduler is based on requesting, booking and scheduling services, like examinations, tests, etc. as patient appointments (see Figure 1). These appointments involve different resources within the hospital like equipment, examination rooms, physicians and patients. These resources belong to different organisational units, like the service department or the requesting ward. In principle, anything can be named a resource. In contrast to traditional booking and calendar systems supporting the task of scheduling within the service department, the prototype aims to facilitate a more direct collaboration between the employees at the different wards and service departments. Based on the analysis of the work practices at the ward and service departments, support for collaboration in the Patient Scheduler has been divided into three areas: communication, sharing and planning:

Communication: A request for a patient appointment can be sent to another department, team, or whichever organisational unit set up to receive appointments at the hospital. When received, appointments can be sorted into different intrays (both manually and automatic) and scheduled according to different resource calendars. The status (requested, scheduled, performed, halted, etc.) of each appointment is generally accessible for inspection.

Planning: When requesting future examinations of a patient a deadline can be added to the request, indicating the latest acceptable time for examination. If the service department cannot comply with this deadline, a message can automatically be routed back to the sender on his request. Furthermore, the tool supports the creation of an examination programme (see Figure 1) consisting of several templates for patient appointments. Such a programme could be an unravelling programme and can be built up in the process of using the Patient Scheduler. A patient appointment can at any time be made into a template 
and added to a programme. These programmes and templates are in return available for use within the department (organisational unit) and can be instantiated on a particular patient. When instantiating a template or a programme the user can modify the resulting appointment(s) before sending it (them) to a recipient. Unnecessary appointments, e.g. the hip examination, can be skipped if desired.

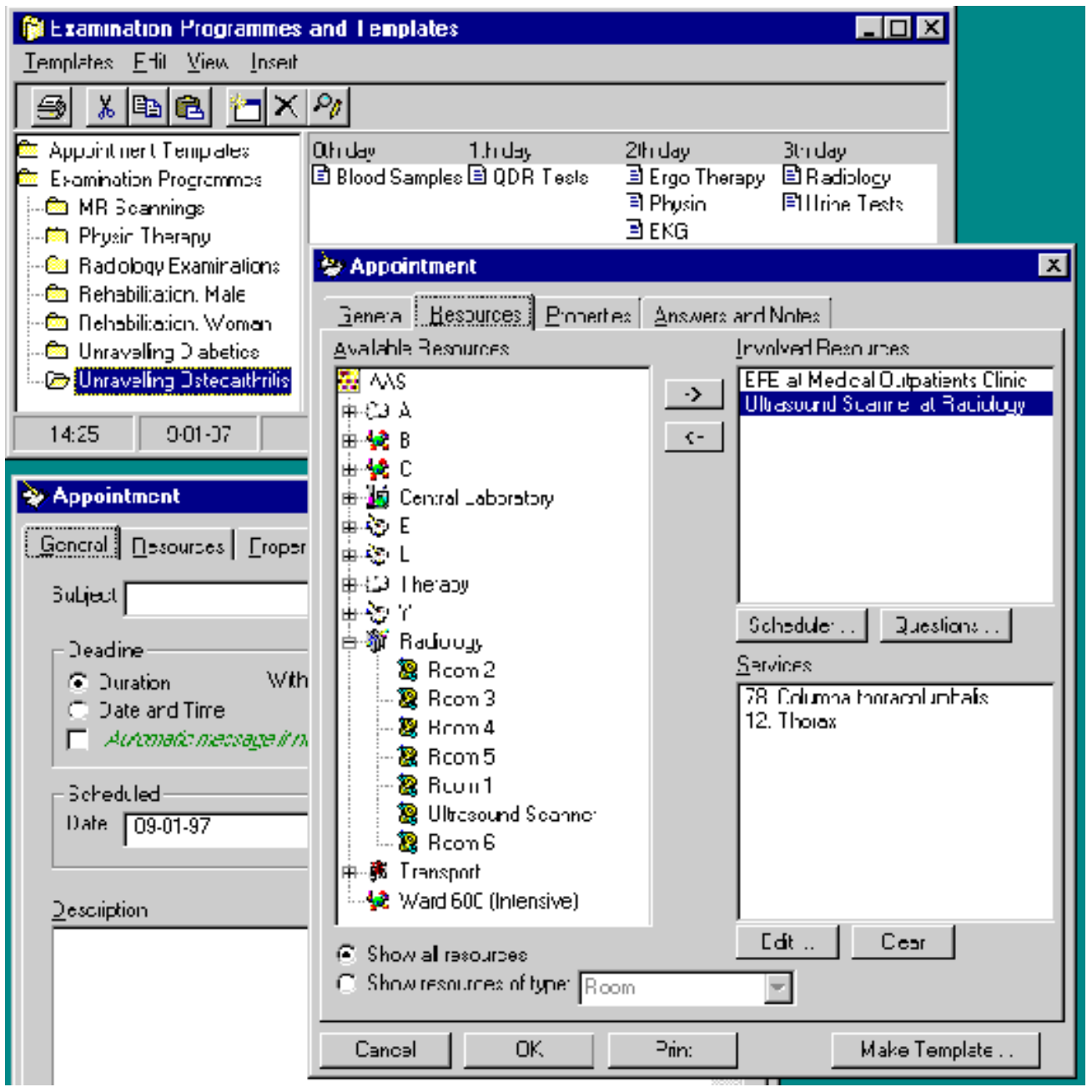

Figure 1: The Examination Programmes and an Appointment involving several resources.

Sharing: The sharing mechanism makes the scheduled appointments accessible within the hospital. By looking into this shared pool of appointments, the Patient Scheduler can generate different comprehensive views on patient appointments e.g. a view on appointments involving a certain department, ward or physician; day calendars showing appointment with the CT-scanner ; and, most important, a shared calendar for each patient at the hospital. This shared patient calendar gives an overview of the status of the patient s trajectory and enables the users to schedule the treatment of the patient according to the patient $\mathrm{s}$ other appointments. The different service departments, like radiology, can share (part of) their resource calendars, hence enabling other departments to directly book trivial examinations that need no approval from a radiologist. This opens up for considerable timesaving in the daily routine examinations. Finally, appointment templates and examination programmes can be shared enabling e.g. the ward to use templates and programmes made at the radiology department. 


\section{Rethinking Workflow as Situated Planning}

A typical workflow system helps to define, execute, coordinate and monitor the flow of work within an organisation. In order to do this a workflow system must contain a computerised representation of the structure of the work procedures and activities. Such a computerised representation has often been a sequential or hierarchical decomposition of an activity into tasks and are built separate to the execution of the activity. As stated by Schäl (1996):

"Workflow management technology is composed of a workflow modelling component and a workflow execution component. The workflow modelling component enables administrators, users and organisational analysts to define working processes, so that processes and activities are defined, analysed, simulated and allocated to people (roles)" (p. 90)

These computerised representations cannot take into account unforeseen events and breakdowns. The decomposition into tasks builds on several assumptions concerning the conditions of future work and the typical problems with a workflow system arise when these assumptions break down. Hence, exception handling has attained considerable attraction within workflow management technologies, and questions on how to handle unforeseen situations and how to design for unanticipated use are often raised. The central point of this paper, however, emphasises that breakdown situations are not exceptions from work activities but are a natural and very important part of any activity which forms the basis for learning and thus for developing and enhancing plans for future action. When synthesised with the current conditions, the plan is a central resource in the realisation of any activity and is subsequently enhanced based on the experience obtained during this activity. Of course, it is important to consider exactly who is allowed to use, alter and save plans within a work practice, but this is a question of division of work and corresponding access rights within the computer system not a separation of the planning and execution of work.

\section{A New Understanding of Plans Based on Activity Theory}

Based on Activity Theory a plan can be defined as a cognitive or material artifact which supports the anticipatory reflection of future goals for actions, based on experience about recurrent structures in life. As an artifact, the plan is socially constructed, is eventually crystallised into a material form, is shared among the actors in the work practice, is used to mediate work, and constitute a central part of the organisation s material conditions for work. A plan is a series of expectations to future results under certain conditions and the execution becomes an afferent synthesis between the plan and the conditions of the concrete situation. The fundamental feedback loop in the course of an activity forms the basis for a learning process embedded in the activity. This learning process creates and enhances the plan, which was originally the guiding principle for the activity.

\section{Characteristics of Computer Tools Supporting Planning}

According to the above understanding of planning as a central part of human activity, a major challenge for planning tools is to support the anticipation of recurrent events in working life and in turn to use this anticipation in the course of work. Based on this conceptualisation of human activity some characteristics of computer support for planning can be drawn from our analysis of medical work and from designing the Patient Scheduler. These characteristics can be read as guidelines for design. 


\section{Producing and Altering Plans in the Course of Work}

The experience of using a plan to guide an activity under certain conditions is obtained during the activity itself. So, in order for plans to become resources for the future realisation of an activity, the plan should be made as part of this activity situated planning. Thus, it is important that the planning tool allows for the ongoing creation and modification of a plan based on obtained experience in realising the plan. The Patient Scheduler supports this in a simple way by allowing any appointment, expected to be used in the future, to be transformed into a template and added to an examination programme. These examination programmes can in turn be modified by sharing, moving and copying templates within and between programmes.

\section{Sharing Plans Within a Work Practice}

The use of the 24-hour-care plan at the ward illustrates how central the sharing of plans are, when they are used as coordination mechanisms among several actors involved in an activity. When all involved personnel has access to use the shared plan, the need for communication is considerably reduced. This enables the involved actors to act as a collective subject with a common motive. In the Patient Scheduler the underlying access mechanism controls who has access to plans enabling plans to be shared among employees and/or departments at the hospital.

\section{Executing Plans According to the Conditions of the Work}

The difference between plans as anticipated results of actions and the realisation of these actions as operations according to the conditions of the situation should be considered when designing a planning tool. Because anticipation will always be imperfect any instantiation of a plan should be malleable. For example, in the Patient Scheduler every appointment made on the basis of an examination programme can be altered or skipped according to the need of the user.

\section{Inspecting Plans and their Potential Outcome}

First of all, an overview of the available planning artifacts within a work practice is clearly a prerequisite for using plans in the first place. The Patient Scheduler supports this in the examination programme window (Figure 1). Secondly, to avoid pure trial-and-error use of plans, the tool must reveal the potential outcome from applying a particular plan. This can be accomplished in many ways. In the Patient Scheduler, the appointment templates within a programme are listed according to a time axis, revealing, in a rudimentary fashion, the temporal order of the resulting appointments from applying the plan. As discussed at AAS, another way of revealing the result of instantiating a plan, is a simulation mechanism: being able to simulate the plan and alter the resulting scheduling of patient appointment, before letting them loose within the hospital. This simulation part of the prototype has not yet been implemented. Finally, the overview of plans should reveal the condition under which the plan is useful and helps establish whether some concrete conditions match the conditions of the plan. This is supported in a very rudimentary way in the Patient Scheduler, where an examination programme contains a textual description of the premises of the plan, leaving it to the user to establish the connection between this description and his current conditions.

\section{Monitoring the Execution of Plans}

Having an overview of the unfolding of activities is essential to all work. However, when the work is 
initiated on the basis of a plan, it becomes important to monitor the progress in work according to the plan. Thus, recognising any deviation from the plan is particularly important and should be supported by the planning tool. This monitoring of any deviation from a plan also encompasses any initial deviation when instantiating the plan, as emphasised in the above guideline. This part has not yet been implemented in the Patient Scheduler. When the user has instantiated an examination programme the resulting appointments cannot be traced backward to the original programme. This functionality, however, was raised and discussed as a central requirement during several prototyping sessions.

\section{Conclusion: Plans as Situated Actions or Technologies of Accountability}

This paper has re-entered into the discussion on how to support ways of planning and prescribing work by providing a new conceptualisation of the role of plans and prescriptions in work activities. By analysing the work within a hospital and designing computer support for planning work, it was illustrated that planning is not to be viewed as opposed to work in situ. Plans as chains of anticipated goals, are a central part of human activity, but are realised accommodated to the contextual conditions. The core point is to recognise the function of plans as ways of anticipating and pre-handling events in (working) life based on their recurrent nature, and be able to save and later reuse the experience obtained in handling these events. Winograd and Flores (1986) make the same argument by showing how many patterns of action within organisations are designed to anticipate and cope with such recurrent structures. This is especially evident within a hospital; plans for handling all kinds of recurrent events, from receiving injured people involved in car accidents to ordering food for patients at the ward daily, have been made and constitute the operational backbone of the hospital. This understanding of plans as central assets in work has some implication for the issue of workflow systems: instead of supporting routing information around in organisations according to a workflow process model, the computer should be a tool mediating the anticipatory reflection of recurrent events in working life. Hence, such a planning tool should support situated planning building, altering, sharing, executing, and monitoring plans within the cooperative work activities.

Based on this conceptualisation it becomes possible to make a planning tool that does not emphasise a rigid match between process models and work. However, it is central to understand why such formal process models are made and embedded in workflow systems in the first place. Often e.g. in the area of Business Process Reengineering workflow systems are viewed as the enabling technologies for turning the modern firm into a process organisation with greater opportunities for efficiency and cost reduction (see e.g. Abbott and Sarin, 1994). Thus, workflow systems are conceived as organisational infrastructure used and designed for meeting organisational goals (e.g. customer satisfaction) (Schäl, 1996). When viewed from this overall organisational perspective, workflow systems are often used to keep track of the work according to these organisational goals. This means that a workflow system is not just mediating the workflow (which has been the premise for this paper so far), but is used for additional managerial purposes. Hence, the workflow system becomes a technology of accountability as defined $b$ : Suchman (1994):

"By technologies of accountability I mean systems aimed at the inscription and documentation of actions to which parties are accountable [...] in the sense represented by the bookkeeper s ledger, the record of accounts paid and those still outstanding" (p. 188).

In this sense the actions realised by the workflow system are polymotivated. On the one hand, the 
system is used to give order to the unfolding of work within the organisation by making some top-down decomposition of the organisational goals into work processes. On the other hand, the system is a technology of accountability by recording the progress of work according to such process models.

The idea of many workflow systems is to consider this polymotivated nature of organisational work and try to integrate (at least) these two motives within the organisation in one system. Unfortunately, this often ends up in having the organisational and administrative activities setting the agenda for the work activities. For example, Bowers et al., (1995) describe a workflow system that embeds the motive of management of keeping track of print-work at the expense of the motive of the employees at the shopfloor of maintaining a smooth flow of work . Similarly, Heath and Luff (1996), reporting from a case study in the Healthcare sector in the UK, illustrate how a workflow system is designed to satisfy the motive of the pharmaceutical firms to record the amount of used medication, at the expense of the motive of the medical practitioners to structure their medical record according to descriptive economies

The point to be emphasised here is that such problems with existing workflow systems should not be understood merely as conflicting motives and goals within the organisation which could easily end up in a conclusion saying that either you design for accountability or you design for work support. It is important to recognise that an organisation, like a hospital, is not merely getting the work done, e.g. curing patients, but is doing this work in a visible, inspectable, documentable and accountable way (Bowers et al., 1995). An organisation is not only engaged in the activity of producing a product, or curing patients. An organisation has to be viewed as a collection of multiple activities, each realising different needs. Some of these activities are directed toward the object of the organisation, like curing patients, and others are directed toward an organisational accountability of work. From an Activity Theory perspective this means that the polymotivated nature of actions involved in a plan should be considered so that motives of all involved actors, responsible for different areas of the work within the organisation, are recognised and satisfied if possible.

\section{Acknowledgements}

Thanks to the employees at AAS and to Trine Grundahl with whom the workplace studies were done and discussed.

\section{References}

Abbott, K., and Sarin, S. (1994): "Experiences with workflow management: Issues for the next generation", In
Proceedings of the Conference on CSCW, Chapel Hill, USA. ACM, p. 113-120.

Anokhin, P. K. (1973): "The forming of natural and artificial intelligence", Impact of Science on Society XXIII (3).

Anokhin, P. K. (1976): "The Philosophical Importance of the Problem of Natural and Artificial Intellects", Soviet Studies in Philosophy XIV (4), p. 3-27.

Bærentsen, K. (1989): "Mennesker og Maskine [Man and Machine]", In Hedegaard, Hansen, and Thyssen (eds.): Et virksomt liv [An active life]. Aarhus: Aarhus Universitets Forlag, p. 142-187.

Bødker, S. (1991): Through the Interface: A Human Activity Approach to User Interface Design. Hillsdale, NJ: LEA.

Bowers, J., Button, G., and Sharrock, W. (1995): "Workflow from within and without: Technology and Cooperative Work on the Print Industry Shopfloor", In Proceedings of the Fourth European Conference on CSCW, Stockholm, Sweden. Kluwer Academic Publishers, p. 51-66. 
Heath, C. and Luff, P. (1996): "Documents and Professional Practice: bad organisational reasons for good clinical records", In Proceedings of the Conference on CSCW, Boston, Massachusetts USA. ACM, p. 354-363.

Hughes, J. and King, V. (1993): "Paperwork", In S. Benford and J. Mariani (Eds.): COMIC D4.1: Requirements and Metaphors of Shared Interaction, Lancaster: Lancaster University.

Kuutti, K. (1991): "The concept of activity as a basic unit of analysis for CSCW research", In Proceedings of the Second European Conference on CSCW, Amsterdam. Kluwer Academic Publisher, p. 249-264.

Leontjev, A. (1978): Activity, Consciousness, and Personality, Englewood Cliffs NJ: Prentice-Hall.

Leontjev, A. (1981): Problems of the Development of Mind. Moscow: Progress Publishers.

Medina-Mora, R., Winograd, T., Flores, R. and Flores, F. (1992): "The action workflow approach to workflow management", In Proceedings of the Conference on CSCW, Toronto, Canada. ACM, p. 281-288.

Nardi, B. A., (ed.) (1996): Context and Consciousness: Activity Theory and Human-Computer Interaction. Cambrigde, MA: MIT Press.

Schäl, T. (1996): Workflow Management Systems for Process Organisations. Berlin: Springer Verlag.

Schmidt, K. and Simone, C. (1996): "Coordination mechanisms: Towards a Conceptual Foundation of CSCW Systems Design", Computer Supported Cooperative Work 5, p. 155-200.

Star, S. L. (1989): "The Structure of Ill-Structured Solutions: Boundary Objects and Heterogeneous Distributed Problem Solving", In L. Gasser and M. Huhns (Eds.): Distributed Artificial Intelligence, London: Pitman, p. 37-54.

Strauss, A., Fagerhaugh, S., Suczek, B. and Wiener, C. (1985): Social Organization of Medical Work. Chicago and London: University of Chicago Press.

Suchman, L. (1987): Plans and situated actions. The problem of human-machine communication. Cambridge: Cambridge University Press.

Suchman, L. (1994): "Do categories have politics? The language/action perspective reconsidered", Computer Supported Cooperative Work 2 (3), p. 177-190.

Symon, G., Long, K., and Ellis, J. (1996): "The Coordination of Work Activities: Cooperation and Conflict in a Hospital Context", Computer Supported Cooperative Work 5, p. 1-31.

Vallgårda, S. (1992): Sygehuse og sygehuspolitik i Danmark: Et bidrag til det specialiserede sygehusvaesens historie 1930-1987. [Hospitals and hospital politics in Denmark: A contribution to the history of the specialised hospital sector 1930-1987]. København: Jurist- og Økonomforbundets Forlag.

Vygotskij, L. S. (1978): Mind and Society. Cambrigde, MA: Harvard University Press.

Winograd, T. (1994): "Categories, diciplines and social coordination", Computer Supported Cooperative Work 2 (3), p. $177-190$.

Winograd, T. and Flores, F. (1986): Understanding Computers and Cognition: A New Foundation for Design. Norwood, New Jersey: Ablex Publishing Corp. 